\title{
ESTANDARIZACION DEL BIOENSAYO DE TOXICIDAD AGUDACON DIPLODON CHILENSIS USANDO UN TOXICO DE REFERENCIA
}

\author{
STANDARIZATION OF THE ACUTE TOXICITY BIOASSAY WITH \\ DIPLODON CHILENSIS USING A REFERENCE TOXICANT
}

\author{
Jeannette Silva ${ }^{1}$, Carmen Fuentealba ${ }^{1}$, Enrique Bay-Schmith ${ }^{1} \&$ Alberto Larrain ${ }^{1}$ \\ ${ }^{1}$ Laboratorio de Bioensayos, Departamento de Zoología, Facultad de Ciencias Naturales y Oceanográficas, \\ Universidad de Concepción, Chile. Email: jsilva@udec.cl
}

\begin{abstract}
RESUMEN
El amplio rango de sensibilidades de las diferentes especies de organismos a la extensa diversidad de contaminantes ambientales hacen recomendable utilizar una batería de especies distintas en las evaluaciones de toxicidad ambiental. Los bioensayos con cada una de esas especies son estandarizados para minimizar las variaciones en mediciones sucesivas. El bivalvo dulceacuícola Diplodon chilensis es un potencial organismo experimental para bioensayos de toxicidad en base a sus poblaciones abundantes y de amplia distribución en ríos y lagos de Chile y por su trascendencia ecológica para las comunidades planctónicas por ser un poderoso filtrador. Se describe un procedimiento de bioensayo estandarizado con $D$. chilensis seguido de la calibración del método. Juveniles de esta especie fueron expuestos por $96 \mathrm{~h}$ a una serie de concentraciones del tóxico de referencia, dicromato de potasio $\left(\mathrm{K}_{2} \mathrm{Cr}_{2} \mathrm{O}_{7}\right)$. El efecto cuantificado fue la mortalidad de los organismos. De un total de ocho estimaciones de la concentración letal $50\left(\mathrm{CL}_{50-96 h}\right) \mathrm{se}$ obtuvo un valor promedio de $20,4 \mathrm{mg} \mathrm{L}^{-1}$ con una desviación estándar (ds) de 3,3 $\mathrm{mg} \mathrm{L}^{-1}$. Se determinó, además, la precisión intralaboratorio del bioensayo con el tóxico de referencia mediante la estimación del coeficiente de variación $(\mathrm{CV}=16,2 \%)$ y el rango dentro del cual se deberían encontrar futuros valores de la $\mathrm{CL}_{50-96}$ para la especie con este tóxico de referencia de acuerdo con la carta de vigilancia. Los límites de confianza superior (LCS) e inferior (LCI) de la $\mathrm{CL}_{50}$ al $95 \%$ de confianza son 27 y $17,1 \mathrm{mg} \mathrm{L}^{-1}$, respectivamente.
\end{abstract}

PALABRAS Claves: Bivalvos dulceacuícolas, calibración, coeficiente de variación, dicromato de potasio.

\section{ABSTRACT}

A wide range of species vulnerability to toxicans makes necessary to use a combination of different species in environmental toxicity assessment. Species specific bioassays are standarized to minimize variations in successive measurements. The freshwater bivalve molluscs Diplodon chilensis is a potential test species for toxicity bioassay based on its widespread and abundant populations in rivers and lakes of Chile and its ecological significance in the plancktonic community as a powerful filter feeder. A standardized procedure with $D$. chilensis is herein described followed by the method's calibration. Juveniles of this species were exposed to a concentration gradient of potassium dichromate $\left(\mathrm{K}_{2} \mathrm{Cr}_{2} \mathrm{O}_{7}\right)$ as reference toxicant for $96 \mathrm{~h}$. The measured effect was mortality. After eight successive estimations of the median lethal concentration $\left(\mathrm{LC}_{50-96 \mathrm{~h}}\right)$ a mean value $\pm \mathrm{SD}$ of $20,4 \pm 3,3 \mathrm{mg} \mathrm{L}^{-1}$ was found. Intra laboratory precision, estimated by means of the variation coefficient (VC), was $16,2 \%$ and the superior and inferior control limits for future LC50 values derived from the control chart were 27 and $17,1 \mathrm{mg} \mathrm{L}^{-1}$ respectively. This bivalve can then be used as test organism in ecotoxicity assessment increasing the diversity of species in environmental testing batteries, with the extra benefit of being a mollusc, zoological group poorly represented among freshwater testing animals in ecotoxicological bioassays.

KEYwords: Freshwater bivalves, calibration, variation coefficient, potassium dichromate. 


\section{INTRODUCCION}

La liberación de sustancias tóxicas en los ecosistemas acuáticos produce una variedad de respuestas complejas en los organismos, las que precisan ser evaluadas. Para ello se han implementado los bioensayos, que son técnicas de evaluación de los efectos tóxicos agudos o crónicos, tanto de sustancias químicas conocidas como de muestras ambientales de composición incierta. Estas pruebas de toxicidad tienen como objetivo medir el efecto de uno o más contaminantes sobre las especies y consiste en la exposición de los organismos de ensayo a concentraciones crecientes de un agente tóxico con el objetivo de determinar algún cambio en éstos en un cierto período de tiempo (Larrain 1995).

Al implementar los bioensayos de toxicidad, es necesario efectuar su estandarización, que consiste en establecer una metodología común en relación, tanto a los parámetros de cultivo (reclutamiento, mantención y cultivo masivo de los organismos) como a los experimentales, que aseguren la reproducibilidad del experimento permitiendo comparar los resultados generados. Diversas organizaciones internacionales (e.g., USEPA, CEE, ASTM CETESB, ISO) han estandarizado metodologías para la realización de estos bioensayos con distintas especies, en donde además se describen métodos de cultivo, condiciones de los experimentos, ámbito de aplicación y restricciones. Es recomendable que cada país tenga estandarizado diversos métodos de ensayo donde las especies autóctonas estén, en lo posible, representadas. En Chile, el bioensayo con el cladócero Daphnia obtusa, una especie nativa, ha sido estandarizado por Silva et al. (2003) por ventajas de sensibilidad y prolificidad respecto de las especies Daphnia magna y Daphnia pulex que actualmente conforman la norma chilena para determinación de toxicidad aguda con este tipo de organismos (NCh 2083). En nuestro país se han utilizado varias especies autóctonas como organismos de ensayo en evaluaciones de toxicidad tales como el erizo de mar Arbacia spatuligera (Larrainet al. 1999), el anfipodo marino Ampelisca araucana (Larrain et al. 1998), el pejerrey Odontesthes regia (Silva et al. 2001) y los copépodos Tisbe longicornis (Larrainet al. 1998) y Eucyclops neumani neumani (Soto et al. 2003). Algunas especies de bivalvos marinos como Argopectenpurpuratus (Troncosoetal.2000), Aulacomya ater, Choromytilus chorus, Perumytilus purpuratus y Semimytilus algosus (Zúñiga et al. 2003) también han sido usadas como organismos de ensayo. Para el ambiente acuático continental de Chile no se dispone de un protocolo estandarizado de bioensayo de ecotoxicidad que incorpore a moluscos como especie de prueba.

Los bivalvos dulceacuícolas han sido señalados como buenos indicadores de contaminación por sus hábitos sedentarios y su longevidad. Además resisten prolongados períodos de inanición y baja oxigenación, pudiendo mantenerse fácilmente en condiciones de cautiverio (Busse 1970). Diplodon chilensis, conocido como chorito de agua dulce, es un bivalvo de amplia distribución en lagos y ríos de Chile (Parada \& Peredo 2006), al cual se le atribuye, por su tamaño y hábito filtrador, una influencia determinante en las comunidades fitoplanctónicas de esos ambientes (Lara \& Parada 1991, Soto \& Mena 1999). En este trabajo se evalúa la potencialidad de emplear a $D$. chilensis como organismo de ensayo, frente al tóxico de referencia que se ha usado preferentemente en todas las especies indicadas anteriormente, dicromato de potasio $\left(\mathrm{K}_{2} \mathrm{Cr}_{2} \mathrm{O}_{7}\right)$ Una vez estandarizada la metodología experimental del bioensayo de toxicidad aguda, se realiza la calibración del método. La calibración es un proceso cuyo objetivo es determinar la precisión y exactitud que debe alcanzarse en los resultados generados por un determinado bioensayo. Lo anterior es útil para asegurarse que la respuesta de la población expuesta a un xenobiótico se deba al efecto de éste y no a variaciones tanto de la sensibilidad de los organismos como a fallas operacionales en la aplicación del método. En este estudio se evalúa la sensibilidad de $D$. chilensis respecto al $\mathrm{K}_{2} \mathrm{Cr}_{2} \mathrm{O}_{7}$ y se define el rango de variabilidad máximo aceptable en los resultados generados por el bioensayo con el tóxico de referencia.

\section{MATERIALES Y METODOS}

Diplodon chilensis es una almeja común en lagos y ríos del centro-sur de Chile y Argentina. En Chile se distribuye desde el río Mataquito - Curicó (VII Región) (3458' S; 71²4'W) hasta el río Negro - 
Itsmo de Ofqui (XI Región) (46³7’ S; 74¹0’W) en tanto que en Argentina se distribuye desde

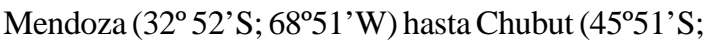
$\left.67^{\circ} 28^{\prime} \mathrm{W}\right)$. Posee gran capacidad de filtración, lo que lo hace atractivo en el tratamiento biológico de aguas servidas. D. chilensis tiene un alto valor ecológico, ya que su actividad filtrante puede mantener en equilibrio cuerpos de agua que reciben cargas de material orgánico. Tienen la capacidad de colonizar sustratos arenosos, areno-pedregosos y fangosos alcanzando densidades variables y crecientes desde $10 \mathrm{ind} / \mathrm{m}^{2}$ en sustratos arenosos a $361 \mathrm{ind} / \mathrm{m}^{2}$ en sustratos fangosos (Lara et al. 2002).

LUGAR DE MUESTREO

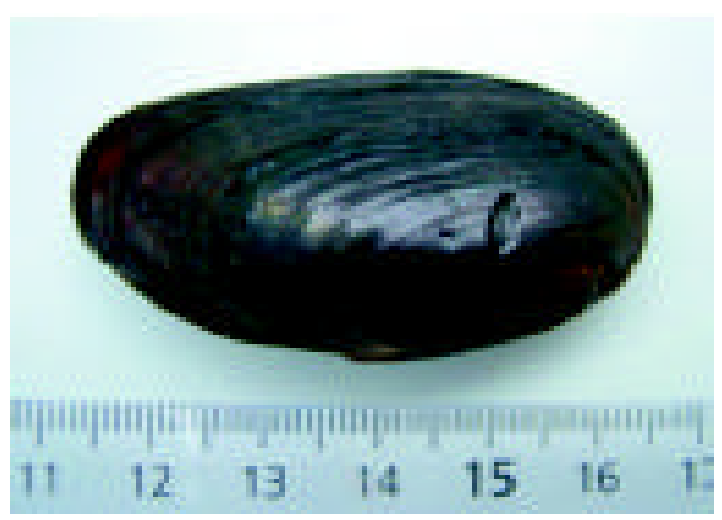

Figura 1. Diplodon chilensis (Gray 1828).

FIGURE 1. Diplodon chilensis (Gray 1828).

Todos los ejemplares fueron extraídos de la Laguna Chica de San Pedro (36 $51^{\circ}$ ” S; $73^{\circ}$ 05” W, VIII Región, Chile), un sistema léntico mesotrófico que no recibe descargas industriales o domésticas.

\section{Procedimiento EXPERIMENTAL}

Como organismos de ensayo se eligieron bivalvos de una talla entre 5,5 y $10 \mathrm{~cm}$, los cuales fueron aclimatados por al menos 1 semana en acuarios de $80 \mathrm{~L}$ con agua potable declorada y aireación permanente. Se alimentaron diariamente con microalgas de agua dulce (Scenedesmus spinosus y Selenastrum capricornutum).
Previo al bioensayo definitivo se realizaron los ensayos preliminares para determinar el rango de concentraciones que generan respuestas con y sin efecto letal. Las pruebas definitivas se efectuaron con 5 concentraciones en progresión geométrica más un control.

El gradiente de concentración se preparó a partir de una solución madre de Dicromato de potasio de $10.0000 \mathrm{mg} \mathrm{L}^{-1}$, la que fue diluida para obtener concentraciones de 7,7; 12,9;21,6; 36 y $60 \mathrm{mg} \mathrm{L}^{-1}$, con un volumen final de $2 \mathrm{~L}$ por unidad experimental. La exposición al tóxico fue por 96 h, con renovación de medio a las 48 horas. El bioensayo se mantuvo a una temperatura constante de $20^{\circ} \mathrm{C}$. El agua utilizada para preparar las distintas concentraciones y el control fue agua potable declorada, previamente aireada.

En la evaluación de la respuesta (mortalidad) se usó el criterio de postrelajación valvar y músculo abductor, estimulando la reacción de cierre valvar con una aguja. Con los datos de mortalidad se estimó la Concentración Letal Media $\left(\mathrm{CL}_{50}\right)$ mediante el programa Probit (US EPA 1993).

\section{CAlibración InTRALABORATORIO}

La calibración intralaboratorio del bioensayo con D. chilensis se realizó con un total de 8 repeticiones, entre agosto del año 2004 y abril del 2005, en el Laboratorio de Bioensayos de la Universidad de Concepción, Chile. El número de resultados consecutivos contemplados se ajusta a lo recomendado por USEPA (1993), organismo que establece para una correcta intracalibración un número mínimo de 5 bioensayos triplicados consecutivos. La precisión intralaboratorio fue determinada mediante el cálculo (USEPA 1993) del coeficiente de variación CV (\%) usando la siguiente expresión:

$$
C V(\%)=100 \times \frac{\text { 'd.e }}{!} \overline{\bar{X}},
$$

Donde: $\bar{X}=$ promedio correspondiente a $\operatorname{los} n$ valores de $\mathrm{CL}_{50-96 \mathrm{~h}}$ generados a partir de $\operatorname{los} n$ bioensayos considerados en la calibración

d.e. = desviación estándar 
Mientras más cerca de cero (0) se encuentre el valor para el CV, mayor será la precisión. El Servicio de Protección Ambiental de Canadá (EPS 1990) indica que la mínima precisión intralaboratorio permitida esta representada por un CV $=30 \%$. La Carta de Vigilancia es la herramienta de registro que brinda los elementos de juicio para establecer los intervalos aceptables de variación de la respuesta de los organismos de ensayo a un tóxico de referencia, con un margen de confianza de un $95 \%$. Los valores de $\mathrm{CL}_{50}$ se disponen en una gráfica, la carta de vigilancia, para evidenciar el control de la sensibilidad de la especie empleada, de la estabilidad de la respuesta biológica y de la repetitibidad (exactitud) de los resultados obtenidos siempre y cuando la variabilidad (CV) sea menor o igual al 30 $\%$ (Castillo 2004). La carta de vigilancia generada con los ocho ensayos del bivalvo expuesto a $\mathrm{K}_{2} \mathrm{Cr}_{2} \mathrm{O}_{7}$ se presenta en la Figura 2. Estos valores fueron confrontados, en el mismo gráfico, con la media para los 8 valores de $\mathrm{CL}_{50}$ (línea de tendencia central; LTC) y los límites de confianza superior (LCS) e inferior (LCI) al $95 \%$ para este conjunto de datos.

A partir del quinto bioensayo se comienza a determinar el CV y a construir la Carta de Vigilancia. Cada nuevo resultado $\left(\mathrm{CL}_{50-96 \mathrm{~h}}\right)$ fue comparado con la Carta de Vigilancia existente y si estuvo comprendido dentro de las líneas de vigilancia fue incluido en el cálculo de la misma. El rango LCI-LCS al $95 \%$ es representativo de la sensibilidad de la especie al tóxico de referencia.

\section{RESULTADOS}

VALORES DE $\mathrm{CL}_{50-96 \mathrm{~h}}$ PARA DiCROMATO DE POTASIO EN BIOENSAYOS DE TOXICIDAD CON D. CHILENSIS

Los datos de las $\mathrm{CL}_{50-96 \mathrm{~h}}$ y sus límites de confianza inferior (LCI) y superior (LCS) estimados de los bioensayos de toxicidad aguda de $D$. chilensis con el tóxico de referencia dicromato de potasio $\left(\mathrm{K}_{2} \mathrm{Cr}_{2} \mathrm{O}_{7}\right)$ se presentan en la Tabla I.

\section{ESTADÍSTICOS DESCRIPTIVOS}

Algunos valores de estadísticos descriptivos, como promedio, desviación estándar, coeficiente de variación, quartiles superior e inferior, curtosis y rango intermedio se señalan en la Tabla II. La $\mathrm{CL}_{50-96}$ promediada de los 8 bioensayos fue de $20,4 \mathrm{mg} \mathrm{L}^{-1}$ con un coeficiente de variación (C.V.) de $16,2 \%$, lo cual indica una variabilidad inferior al máximo permitido (C,V d•30\%) por EPS (1990) y USEPA (1993).

TABLA I: Toxicidad letal $\left(\mathrm{CL}_{50-96 \mathrm{~h}}\right)$ del dicromato de potasio $\left(\mathrm{K}_{2} \mathrm{Cr}_{2} \mathrm{O}_{7}\right)$ y su rango de confianza para $D$. chilensis.

TABLE I: Lethal toxicity $\left(\mathrm{LC}_{50-96 \mathrm{~h}}\right)$ and confidence range of potassium dichromate $\left(\mathrm{K}_{2} \mathrm{Cr}_{2} \mathrm{O}_{7}\right)$ to $D$. chilensis.

\begin{tabular}{llll}
\hline & & \multicolumn{2}{c}{$\begin{array}{c}\text { Límite de confianza } \\
(\mathrm{pd} \bullet 0,05)\end{array}$} \\
\hline $\mathrm{N}^{\circ}$ & $\mathrm{C}_{150-96 \mathrm{~h}}$ & $\mathrm{LCI}$ & $\mathrm{LCS}$ \\
& $\mathrm{mg} \mathrm{L}^{-1}$ & $\mathrm{mg} \mathrm{L}^{-1}$ & $\mathrm{mg} \mathrm{L}^{-1}$ \\
1 & 18,0 & 11,4 & 23,9 \\
2 & 16,7 & 13,0 & 21,0 \\
3 & 24,9 & 18,6 & 34,8 \\
4 & 19,9 & 16,3 & 24,3 \\
5 & 19,4 & 16,4 & 23,0 \\
6 & 18,9 & 15,5 & 23,0 \\
7 & 26,0 & 21,3 & 32,0 \\
8 & 19,2 & 15,1 & 24,1 \\
\hline
\end{tabular}

TABLA II: Estadísticos descriptivos para las $\mathrm{CL}_{50-96 \mathrm{~h}} \mathrm{de}$ dicromato de potasio como tóxico de referencia en bioensayos de toxicidad aguda con $D$. chilensis.

TABLE II: Descriptive statistics for potassium dichromate $\mathrm{LC}_{50-96 \mathrm{~h}}$ as reference toxicant in acute toxicity bioassays with $D$. chilensis

\begin{tabular}{lcc}
\hline Estadístico & Unidad & Valor \\
\hline Número de Datos $\left(\mathrm{CL}_{50-96 \mathrm{~h}}\right)$ & - & 8 \\
Promedio & $\mathrm{mg} \mathrm{L}^{-1}$ & 20,4 \\
Desviación estándar & $\mathrm{mg} \mathrm{L}^{-1}$ & 3,3 \\
Valor mínimo & $\mathrm{mg} \mathrm{L}^{-1}$ & 16,7 \\
Valor máximo & $\mathrm{mg} \mathrm{L}^{-1}$ & 26,0 \\
Quartil inferior & $\mathrm{mg} \mathrm{L}^{-1}$ & 18,7 \\
Quartil superior & $\mathrm{mg} \mathrm{L}^{-1}$ & 21,2 \\
Rango interquartil & $\mathrm{mg} \mathrm{L}^{-1}$ & 2,5 \\
Curtosis & - & $-0,136$ \\
Coeficiente de Variación & $\%$ & 16.2 \\
\hline
\end{tabular}




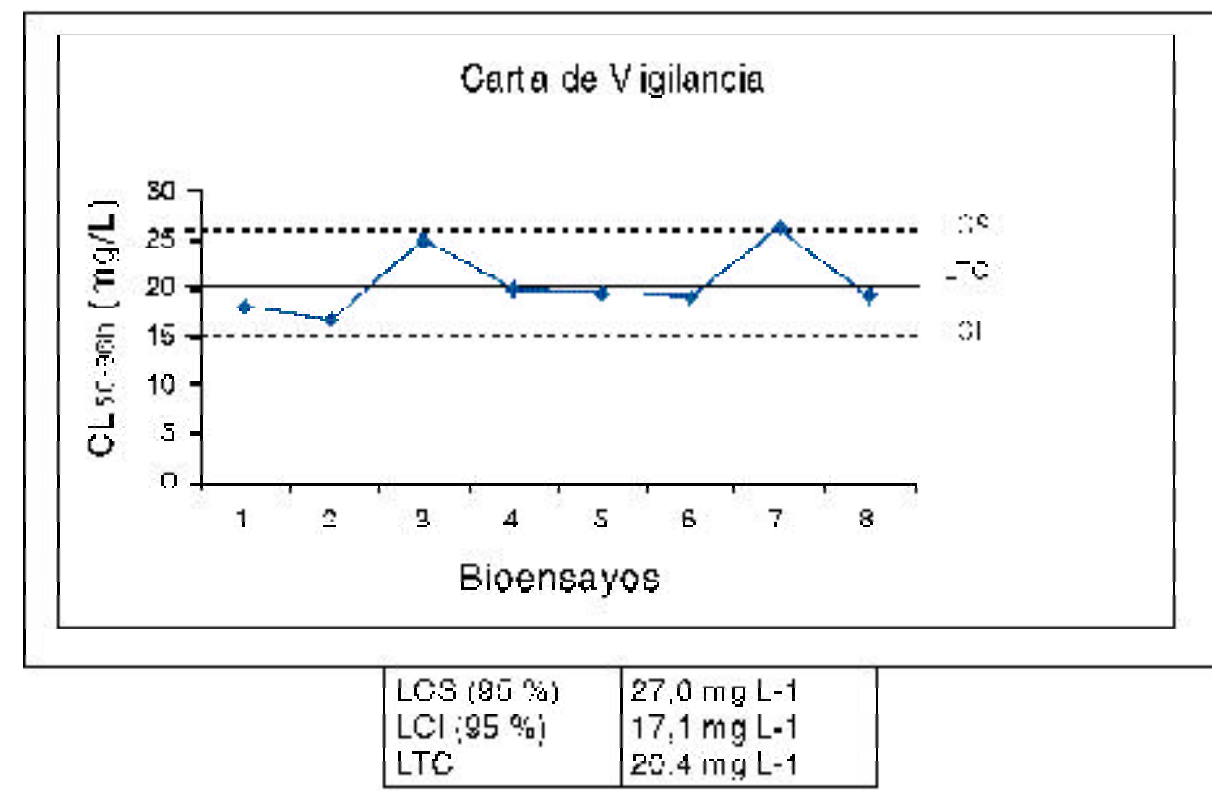

Figura 2: Carta de vigilancia para el bioensayo agudo de Diplodon chilensis con el tóxico de referencia dicromato de potasio.

FIGURE 2: Control chart for Diplodon chilensis acute bioassay with potassium dichromate as reference toxicant.

\section{CARTa de Vigilancia}

La Carta de Vigilancia, confeccionada con los datos $(\mathrm{n}=8)$ obtenidos de la estandarización del bioensayo de D.chilensis con dicromato de potasio, se presenta en la Figura 2. La línea de tendencia central (LTC) representa la $\mathrm{CL}_{50-96 \mathrm{~h}}$ promedio $\left(20,4 \mathrm{mg} \mathrm{L}^{-1}\right)$ cuyos límites superior (LCS) e inferior (LCI) de confianza al $95 \%$ son 27,0 y $17,1 \mathrm{mg} \mathrm{L}^{-1}$, respectivamente.

\section{DISCUSION}

Los bivalvos dulceacuícolas son considerados organismos muy sensibles a la contaminación, por este motivo están desapareciendo de los cursos de agua próximos a zonas urbanas, donde antes eran de gran abundancia. Es así que algunas agencias estadounidenses, como ASTM y EPA, están preocupadas en desarrollar protocolos estandarizados para llevar a cabo bioensayos de toxicidad aguda y crónica con larvas y juveniles de bivalvos dulceacuícolas, por la importancia y vulnerabilidad ecológica en que se encuentran la mayoría de las especies de moluscos en ese país. Uno de estos protocolos, que aún no entra en vigencia (ASTM E2455-05), es para desarrollar bioensayos con larvas glochidio de bivalvos dulceacuícolas.

En Chile se ha observado una importante reducción en la abundancia de la población de Diplodon chilensis en ambientes lóticos. Esta declinación puede ser atribuida a la degradación de la calidad de las aguas, destrucción del hábitat y probablemente a la introducción de peces exóticos que reemplazan a las especies nativas que sirven de huésped para los estados larvales (larva glochidio) (Peredo et al. 2005). La potencialidad de D. chilensis como organismo depurador lo ha hecho objeto de estudios de bioacumulación y filtración (Valdovinos \& Cuevas 1996; Valdovinos et al. 1998). La introducción de este bivalvo en ecosistemas 
eutroficados ha sido sugerida como posible mecanismo mitigador (Mena 1997).

No hay información en la literatura con respecto a estudios ecotoxicológicos con esta especie ni con otros bivalvos de agua dulce de Chile. Se encontró información para una especie de bivalvo marino Crassostrea virginica (ostra americana) con un valor de $\mathrm{CL}_{50-96 \mathrm{~h}}=10,3 \mathrm{mg} \mathrm{L}^{-1}$ para cromo trivalente (Eisler 1986), el cual es más bajo que el encontrado en este trabajo para $D$. chilensis con cromo hexavalente $\left(\mathrm{CL}_{50-96 \mathrm{~h}}=20,4 \mathrm{mg} \mathrm{L}^{-1}\right)$. La sensibilidad de $D$. chilensis al dicromato de potasio es un orden de magnitud más baja que la de otros bivalvos marinos $\left(\mathrm{CL}_{50-96 \mathrm{~h}}\right.$ para Choromytilus chorus es 294 $\mathrm{mg} \mathrm{L}^{-1}$ y para Perumytilus purpuratus es $348 \mathrm{mg} \mathrm{L}^{-1}$ ) (datos no publicados del Laboratorio de Bioensayos, Universidad de Concepción), diferencia probablemente sustentada por una mayor interferencia a la ionización de la sal de cromo en una solución salina como el agua de mar. Obtener estimaciones de su sensibilidad a contaminantes del ambiente acuático contribuirá, por una parte, a precisar los límites de su real capacidad como depurador de aguas contaminadas y, por otra parte, a predecir el riesgo de extinción de sus poblaciones en ambientes de deterioro progresivo.

Tomando en consideración la abundancia de $D$. chilensis en cuerpos de agua continentales, su fácil identificación taxonómica, simple manejo y adecuada aclimatación a las condiciones de laboratorio, sumado a una apreciable sensibilidad al tóxico de referencia y con respuestas reproducibles $(\mathrm{CV}=16,2 \%)$ se puede sugerir su incorporación a las baterías de bioensayos que buscan cubrir el mayor espectro de susceptibilidades metabólicas en las evaluaciones ecotoxicológicas de potenciales contaminantes.

El hábito de vida bentónica, enterrado parcialmente en el sedimento, induce a plantear como hipótesis de futuras investigaciones la validez de esta especie en la evaluación de la calidad de sedimentos de ambientes acuáticos posiblemente alterados.

\section{CONCLUSION}

De este trabajo se desprenden las siguientes conclusiones:

1.- La sensibilidad de la especie Diplodon chilensis al tóxico de referencia (dicromato de potasio) expresada como el promedio de la $\mathrm{CL}_{50-96 \mathrm{~h}}$ de 8 bioensayos consecutivos es $20,4 \mathrm{mg} \mathrm{L}^{-1}$ con una desviación estándar de 3,3 $\mathrm{mg} \mathrm{L}^{-1}$.

2. El límite de vigilancia superior (LCS) e inferior (LCI) de la $\mathrm{CL}_{50-96 \mathrm{~h}}$ del $\mathrm{K}_{2} \mathrm{Cr}_{2} \mathrm{O}_{7}$ es 27,0 y 17,1 mg $\mathrm{L}^{-1}$, respectivamente.

3. La precisión del bioensayo agudo con el chorito de agua dulce, medida como elCV de las $\mathrm{CL}_{50-96 \mathrm{~h}}$ es de $16,2 \%$, valor dentro del rango permitido por EPS (1990) y USEPA (1993).

4. El chorito de agua dulce es un organismo apto para ser utilizado en bioensayos de toxicidad.

\section{BIBLIOGRAFIA}

ASTM E 2455-05. Standard Guide for Conducting Laboratory Toxicity Tests with Freshwater Mussels (actualmente en revisión).

Busse, K. 1970. Nuevo método para medir flujos de agua producidos por organismos filtradores. Medición experimental en Diplodon chilensis (Gray, 1828) (Mollusca, Lamellibranchiata). Notas Mensuales del Museo Nacional de Historia Natural. Santiago. 172:3-10.

Castillo, G. 2004. Ensayos toxicológicos y métodos de evaluación de calidad de aguas. Estandarización, intercalibración, resultados y aplicaciones. (Ed) México: IMTA. 188 pp.

EISLER, R. 1986. Chromium hazards to fish, wildlife and invertebrates: a synoptic review. U.S. Fish and Wildlife Service Biological Report 85 (1.6) 80 pp.

EPS. 1990. Guidance Document on Control of Toxicity Test Precision Using Reference Toxicants. Environment Canada. Report EPS 1/RM/12.

Lara, G. \& E. Parada. 1991. Seasonal changes in the condition index of Diplodon chilensis chilensis (Gray, 1828) in sandy and muddy substrata. Villarrica Lake, Chile (39 $18^{\circ}$ 'S; $\left.72^{\circ} 05^{\prime} \mathrm{W}\right)$. Boletín de la Sociedad de Biología de Concepción 62: 99106.

Lara, G., A. Contreras \& F. Encina. 2002. La almeja de agua dulce Diplodon chilensis (Bivalvia: Iridea) potencial biofiltro para disminuir los niveles de coliformes en pozos: Experimento de laboratorio. Gayana 66(2):113-118.

LARRAIN, A. 1995. Criterios ecotoxicológicos para evaluar alteraciones ambientales y establecer parámetros de control: Importancia de los Bioensayos de Toxicidad. Revista Ciencia y Tecnología del Mar. Cona ( $\mathrm{N}^{\circ}$ Especial):39-47.

Larrain, A., E. Soto \& E. BAy-Schmith. 1998. Assessment of sediment toxicity in San Vicente Bay, Central Chile, using the amphipod Ampelisca araucana. Bulletin of Environmental Contamination and Toxicology 61:363-369. 
Estandarización del bioensayo de toxicidad aguda con Diplodon chilensis: SiLVA, J. ET AL.

Larrain, A., E. Soto, J. Silva \& E. B ay-Schmith. 1998. Sensitivity of the meiofaunal Copepod Tisbe longicornis to $\mathrm{K}_{2} \mathrm{Cr}_{2} \mathrm{O}_{7}$ under varying temperature regimes. . Bulletin of Environmental Contamination and Toxicology 61:391-396.

Larrain, A., A. Riveros, J. Silva \& E. Bay-Schmith. 1999. Toxicity of Metals and Pesticides Using the Sperm Cell Bioassay with the Sea Urchin Arbacia spatuligera Bulletin of Environmental Contamination and Toxicology 62:749-757.

Mena, G. 1997. Evaluación experimental de la capacidad de Diplodon chilensis para procesar los excedentes orgánicos generados por la Salmonicultura. Tesis de Licenciado en Biología Marina. Universidad Austral de Chile. Valdivia, Chile.

NCH 2083 Of.1999 Aguas - Bioensayo de toxicidad aguda mediante la determinación de la inhibición de la movilidad de Daphnia magna o Daphnia pulex (Crustacea, Cladocera). Norma Chilena Oficial NCh 2083. Of1999. Instituto Nacional de Normalización, INN-Chile. (Decreto $\mathrm{N}^{\circ} 152$ )

Parada, E. \& S. Peredo. 2006. Estado del conocimiento de los bivalvos dulceacuícolas de Chile. Gayana 70(1): 82-87.

Peredo, S., E. Parada, I. Valdebenito \& M. Peredo. 2005. Relocation of the freshwater mussel Diplodon chilensis (Hyriidae) as a strategy for its conservation and management. Journal of Molluscan Studies 71: 195-198.

Silva, J., L. Troncoso, E. Bay-Schmith \& A. Larrain. 2001. Utilization of Odonthestes regia (Atherinidae), from the South Eastern Pacific as a test organism for bioassays; study of its sensitivity to six chemicals. Bulletin of Environmental Contamination and Toxicology 66:570-575.
Silva, J., G. Torrejón, E. Bay-Schmith \& A. Larrain. 2003. Calibración del bioensayo de toxicidad aguda con Daphnia obtusa (Crustacea: Cladocera) usando un tóxico de referencia. Gayana 67(1): 87-96.

Soto, D. \& G Mena. 1999. Filter feeding by the freshwater mussel, Diplodon chilensis, as a biocontrol of salmon farming eutrophication. Aquaculture 171: 65-81.

Soto, E., G. Oyarce, B. Inzunza \& E. Bay-Sсhmith. 2003. Acute toxicity of organic and inorganic compounds on the freshwater cyclopoid copepod Eucyclops neumani neumani (Pesta 1927). Bulletin of Environmental Contamination and Toxicology 70:1017-1021.

Troncoso, L., R. Galleguillos \& A. Larrain. 2000. Effects of copper on the fitness of the Chilean scallop Argopecten purpuratus (Mollusca: Bivalvia). Hydrobiologia 420: 185-189.

USEPA. 1993. Methods for measuring the acute toxicity of effluents and receiving waters to freshwater and marine organisms. Office of Research and Development, U.S. Environmental Protection Agency, Washington D.C. 206460. EPA/600/ 4-90/027F.

Valdovinos, C \& R. Cuevas. 1996. Tasas de aclarancia de Diplodon chilensis (Bivalvia, Hyridae): Un suspensívoro bentónico dulceacuícola de Chile Central. Medio Ambiente 13(1):114-118.

Valdovinos, C., R. Figueroa, H. Cid, O. Parra, E. Araya, S. Privitera \& V. Olmos. 1998. Transplante de organismos bentónicos entre sistemas lénticos: ¿Refleja la biodisponibilidad de metales traza en el ambiente? Boletín de la Sociedad Chilena de Química 43:467-475.

Zúñiga, M., P. Vallejos, A. L arrain \& E. B AY-Schmith. 2003. Toxicity of copper on four Chilean marine mussels. Bulletin of Environmental Contamination and Toxicology 71:1167-1174. 